COMMUNICATIONS IN

ANALYSIS AND GEOMETRY

Volume 13, Number 3, 591-631, 2005

\title{
Square numbers, spanning trees and invariants of achiral knots
}

\author{
A. Stoimenow ${ }^{1}$
}

\begin{abstract}
We give constructions to realize an odd number, which can be represented as sum of two squares, as determinant of an achiral knot, thus proving that these are exactly the numbers occurring as such determinants. Later, we study which numbers occur as determinants of prime alternating achiral knots, and obtain a complete result for perfect squares. Using the checkerboard coloring, then an application is given to the number of spanning trees in planar self-dual graphs. Another application is some enumeration results on achiral rational knots.
\end{abstract}

\section{Introduction.}

The main problem of knot theory is to distinguish knots (or links), i.e., smooth embeddings of $S^{1}$ (or several copies of it, called components) into $\mathbb{R}^{3}$ or $S^{3}$ up to isotopy. A main tool for this is to find invariants of knots, i.e., maps of knot diagrams into some algebraic structure, which are invariant under Reidemeister's moves. A family of most popular such invariants are the polynomial invariants, associating to each knot an element in some oneor two-variable (Laurent) polynomial ring over $\mathbb{Z}$. Given a knot invariant, beside distinguishing knots with it, one is also interested in which properties of knots it reflects, and in which way.

One of the most intuitive ways to associate to a knot (or link) $L$ another one is to consider its obverse, or mirror image ! $L$, obtained by reversing the orientation of the ambient space. The knot (or link) is called achiral (or synonymously amphicheiral), if it coincides (up to isotopy) with its mirror image, and chiral otherwise. When considering orientation of the knot, then we distinguish among achiral knots between +achiral and -achiral ones, depending on whether the deformation into the mirror image preserves or

\footnotetext{
${ }^{1}$ Supported by JSPS postdoc grant P04300.
} 
reverses the orientation of the knot. (For links, one has to attach a sign to each component, and take into account possible permutations of the components. Usually, one calls the link + or -achiral, if the orientation of all components is preserved or reverted, without regard to their order, i.e. allowing one component to be mapped to the mirror image of another.)

When the Jones polynomial $V$ [24] appeared in 1984, one of its (at that time) spectacular features was that it was (in general) able to distinguish between a knot and its obverse by virtue of having distinct values on both, and (hence) so were its generalizations, the HOMFLY, or skein, polynomial $P$ [18] and the Kauffman polynomial $F$ [30]. The $V, P$ and $F$ polynomials of achiral knots have the special property to be self-conjugate, that is, invariant when one of the variables is replaced by its inverse. Their decades-old predecessor, the Alexander polynomial $\Delta[2]$, a knot invariant with values in $\mathbb{Z}\left[t, t^{-1}\right]$, was known always to take the same value on a knot and its mirror image. Nevertheless, contrarily to common intuition, $\Delta$ can also be used to detect chirality (the property of a knot to be distinct from its mirror image) by considering its value $\Delta(-1)$, called determinant.

The aim of this paper is to study invariants of achiral knots and to relate some properties of their determinants to the classical topic in number theory of representations of integers as sums of two squares.

After collecting some knot and number theoretic preliminaries in Sections 2.1 and 2.2 resp., we begin in Section 3 with recalling a criterion for the Alexander polynomial of an achiral knot via the determinant, which follows from Murasugi's work on the signature [41] and the Lickorish-Millett [36] value of the Jones polynomial. These conditions show that, paradoxically formulated, although the Alexander polynomial cannot distinguish between a knot and its mirror image, it can still sometimes show that they are distinct.

Then, we show in Section 4.1 that the condition of Section 3 is in fact a reduction modulo 36 of the exact arithmetic description of numbers, occurring as determinants of achiral knots. Namely, an odd natural number is the determinant of an achiral knot if and only if it is the sum of two squares. The 'only if' part of this statement was an observation of Hartley and Kawauchi in [21]. Our aim will be to show the 'if' part, that is, given a sum of two squares, to realize it as the determinant of an achiral knot (theorem 4.1). The main tool used is the definition of the determinant by means of Kauffman's state model for the Jones polynomial [31].

Then, we attempt to refine our construction, by producing achiral knots (of given determinant) with additional properties: prime and/or alternating. Although it turns out that one of these properties can always easily be 
achieved, the situation turns harder when requiring them both simultaneously. We investigate this problem in Section 4.2. Now, the correspondence of Section 4.1 does not hold completely, and there are exceptional values of the determinant, that cannot be realized. To show that 9 and 49 are such, we use a quadratic improvement of Crowell's (lower) bound for the determinant of an alternating knot in terms of its crossing number [9], in the case the knot is achiral (Proposition 4.18). We obtain then a complete result about which perfect squares can be realized as determinants of prime alternating achiral knots (Theorem 4.21).

In Section 4.3, we consider the problem to describe determinants of unknotting number one achiral knots. In this case, the description is even less clear, as we show by several examples.

Then, we give some applications, including enumeration results on rational knots in Section 5, and a translation of the previously established properties to the number of spanning trees in planar self-dual graphs in Section 6.1. As a different application, using the work of Hartley and Kawauchi [21], one can construct many linear recurrent sequences, all of whose odd members are perfect squares, by realizing these numbers as determinants of strongly +achiral knots. A simple example is a particular family of achiral 3-braid links, called "turks head" links (see [26]). By the correspondence between links and graphs, our particular sequence is found enumerating spanning trees in certain "wheel" graphs. (Another, although unrelated, occurrence of wheels in knot theory is explored in [3].)

Several open problems are suggested during the discussion throughout the paper. These problems appear to be involved enough already for knots, so that we waived on an analogous study of links (which are the cases covering the even natural numbers). For links, the unpleasant issue of component orientations also becomes relevant.

\section{Basic preliminaries.}

We briefly clarify most of the basic concepts that will be employed in this paper. (Further and more detailed definitions are still to follow.)

\subsection{Knots and links.}

In the following, knots and links will be assumed oriented, but sometimes orientation will be irrelevant.

Henceforth, $D_{K}$ denotes the double branched cover of $S^{3}$ over a knot $K$, 
associated to the canonical homomorphism $\pi_{1}\left(S^{3} \backslash K\right) \rightarrow \mathbb{Z}_{2}$. By $H_{1}=$ $H_{1}\left(D_{K}\right)=H_{1}\left(D_{K}, \mathbb{Z}\right)$, we denote its homology group over $\mathbb{Z}$. $H_{1}$ is a finite commutative group of odd order. This order is called the determinant of a knot $K$, and it will be denoted as $\operatorname{det}(K)$. (This generalizes to links $L$, by putting $\operatorname{det}(L)=0$ to stand for infinite $H_{1}\left(D_{L}\right)$.) Its name originates from its expression (up to sign) as the determinant of a Seifert [49, p. 213] or Goeritz [19] matrix.

The Jones polynomial $V$ (introduced in [24], but now commonly used with the convention of [25]) is a Laurent polynomial in one variable $t$ of oriented knots and links, and can be defined by being 1 on the unknot and the (skein) relation

$$
t^{-1} V\left(L_{+}\right)-t V\left(L_{-}\right)=-\left(t^{-1 / 2}-t^{1 / 2}\right) V\left(L_{0}\right) .
$$

Herein $L_{ \pm, 0}$ are three links with diagrams differing only near a crossing.

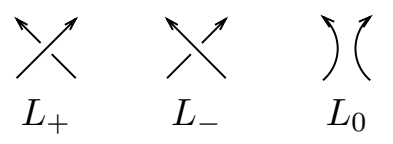

We call the crossings in the first two fragments resp. positive and negative. Replacing $L_{+}$by $L_{-}$(resp. vice versa) is called switching (or changing) a positive (resp. negative) crossing. The sum of the signs $( \pm 1)$ of the crossings of a diagram $D$ is called writhe of $D$ and written $w(D) . w(D)$ is invariant under simultaneous reversal of orientation of all components of the diagram, so is in particular well-defined for unoriented knot diagrams. It may, however, change if some (but not all) components of a link diagram are reverted.

A different interpretation of the Jones polynomial than via skein rules has been developed by Kauffman [31] (see also [1, Section 6.2]). The Kauffman state model is sometimes more useful than the skein approach, and we shall also consider it below. Recall, that the Kauffman bracket $\langle D\rangle$ of a(n unoriented) link diagram $D$ is a Laurent polynomial in a variable $A$, obtained by the sum

$$
\sum_{\text {state } S} A^{\# A-\# B}\left(-A^{2}-A^{-2}\right)^{|S|-1}
$$

Herein a state $S$ is a choice of splittings (or splicings) of type A or B for any single crossing, \#A and \#B denote the number of type A (resp. type B) 
splittings and $|S|$ the number of (disjoint) circles obtained after all splittings in $S$. Splittings are defined as follows:

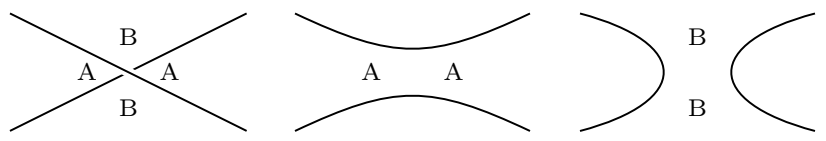

The corner A (resp. B) or a crossing is the one passed by the overcrossing strand when rotated counterclockwise (resp. clockwise) towards the undercrossing strand. A type A (resp. B) splitting is obtained by connecting the A (resp. B) corners of the crossing.

The Jones polynomial of a link $L$ can be calculated from the Kauffman bracket, by evaluating it on the (unoriented version of) a diagram $D$ of $L$, and then multiplying by a power of $t$ coming from the writhe of $D$ :

$$
V_{L}(t)=\left.\left(-t^{-3 / 4}\right)^{-w(D)}\langle D\rangle\right|_{A=t^{-1 / 4}} .
$$

If we modify the skein relation for $V$ by omitting the coefficients $t^{\mp 1}$ of $L_{ \pm}$on the left of (2.1), we obtain the skein relation for another (and more classical) polynomial invariant, the Alexander polynomial $\Delta(t)$. Sometimes, one also uses a topological definition of $\Delta$, which is accurate only up to units in $\mathbb{Z}\left[t, t^{-1}\right]$ (see [49]). Both the Jones and Alexander polynomial allow to express the determinant of $K$, as

$$
\operatorname{det}(K)=\left|\Delta_{K}(-1)\right|=\left|V_{K}(-1)\right| .
$$

The signature $\sigma$ is a $\mathbb{Z}$-valued invariant of knots and links. Originally, defined terms of Seifert matrices [49]. Most of the early work on the signature was done by Murasugi [41], who showed several properties of this invariant. In particular, the following property is known: if $L_{ \pm}$are as in (2.2), then

$$
\sigma\left(L_{+}\right)-\sigma\left(L_{-}\right) \in\{0,1,2\} .
$$

(Note: In (2.5), one can also have $\{0,-1,-2\}$ instead of $\{0,1,2\}$, since other authors, like Murasugi, take $\sigma$ to be with opposite sign. Thus, (2.5) not only defines a property, but also specifies our sign convention for $\sigma$.)

It is known that $\sigma(L)$ has the opposite parity to the number of components of a link $L$, whenever the determinant of $L$ is non-zero (i.e. $H_{1}\left(D_{L}\right)$ is finite). This, in particular, always happens for $L$ being a knot, so that $\sigma$ takes only even values on knots. Therefore, for knots in (2.5) only 0 and 2 can occur on the right. 
The unknotting number $u(K)$ of a knot $K$ is defined as the minimal number of crossing changes in any diagram of $K$ needed to turn $K$ into the unknot (see e.g. $[34,60]$ ). A consequence of the relation $(2.5)$ is the inequality $u(K) \geq|\sigma(K) / 2|$. This is one of the first, and still very valuable, lower unknotting number bounds.

\subsection{Number theory.}

According to a claim of Fermat, written about 1640 on the margins of his copy of Euclid's "Elements", proved in 1754 by Euler, and further simplified to the length of "one sentence" in [65], any prime of the form $4 x+1$ can be written as the sum of two squares. More generally, any natural number $n$ is the sum of two squares if and only if any prime of the form $4 x+3$ occurs in the prime decomposition of $n$ with an even power, and it is the sum of the squares of two coprime numbers if and only if such primes do not occur at all in the prime decomposition of $n$.

The number of representations as the sum of two squares is given by the formula

$$
\begin{aligned}
r_{2}(n): & =\frac{1}{4} \#\left\{\left(m_{1}, m_{2}\right) \in \mathbb{Z}^{2}: m_{1}^{2}+m_{2}^{2}=n\right\}=\sum_{d \mid n}\left(\frac{-4}{d}\right) \\
& =\#\{x \in \mathbb{N}: 4 x+1 \mid n\}-\#\{x \in \mathbb{N}: 4 x+3 \mid n\},
\end{aligned}
$$

which has also an interpretation in the theory of modular forms (see [20, (16.9.2) and theorem 278, p. 275] and [64]).

A number theoretic explanation of (2.6) is as follows: If we denote by $\chi$ the (primitive) character $\left(\frac{-4}{\cdot}\right)$, with -4 being the discriminant of the field of the Gauß numbers $\mathbb{Q}[i]$ and $i=\sqrt{-1}$, we have for $\Re(s)>1$, using that $\mathbb{Q}[i]$ has class number 1 and 4 units, that

$$
\sum_{n=1}^{\infty} \frac{r_{2}(n)}{n^{s}}=\zeta_{\mathbb{Q}[i]}(s)=\zeta(s) L(s, \chi)=\prod_{p \text { prime }} \frac{1}{\left(1-p^{-s}\right)\left(1-\chi(p) p^{-s}\right)}
$$

from which the formula follows by considering the Taylor expansion in $p^{-s}$ of the different factors in the product. (This series converges for $\Re(s)>1$.)

The $\zeta$-function identities also give a formula for

$$
r_{2}^{0}(n):=\frac{1}{4} \#\left\{(a, b) \in \mathbb{Z}^{2}:(a, b)=1, a^{2}+b^{2}=n\right\},
$$

the number of representations of $n$ as the sum of squares of coprime numbers. 
We have

$$
\begin{aligned}
\sum_{n=1}^{\infty} \frac{r_{2}^{0}(n)}{n^{s}} & =\frac{\zeta_{\mathbb{Q}[i]}(s)}{\zeta(2 s)}=\frac{\zeta(s) L(s, \chi)}{\zeta(2 s)} \\
& =\prod_{p \text { prime }} \frac{1-p^{-2 s}}{\left(1-p^{-s}\right)\left(1-\chi(p) p^{-s}\right)}=\left(1+2^{-s}\right) \prod_{\substack{p \equiv 1(4) \\
\text { prime }}} \frac{1+p^{-s}}{1-p^{-s}} .
\end{aligned}
$$

Thus,

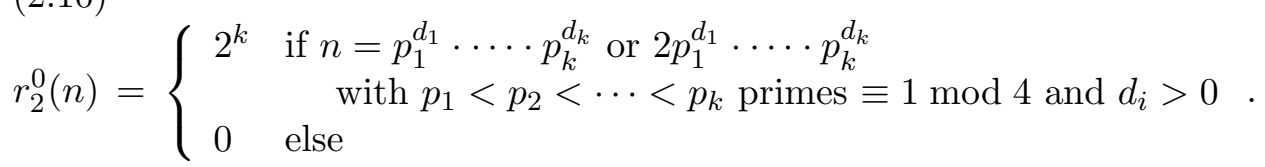

Note, that for $n>1$,

$$
r_{2}^{0}(n)=\#\left\{(a, b) \in \mathbb{N}^{2}:(a, b)=1, a^{2}+b^{2}=n\right\},
$$

and for $n>2$, we have

$$
\frac{r_{2}^{0}(n)}{2}=\#\left\{(a, b) \in \mathbb{N}^{2}:(a, b)=1, a \leq b, a^{2}+b^{2}=n\right\} .
$$

Define the continued fraction for a sequence $\left(a_{1}, \ldots, a_{n}\right)$ of integers:

$$
\left[\left[a_{1}, \ldots, a_{n}\right]\right]:=a_{1}+\frac{1}{a_{2}+\frac{1}{\ldots a_{n-1}+\frac{1}{a_{n}}}} .
$$

Hereby, we use the usual conventions $\frac{1}{0}=\infty, \infty+*=\infty, \frac{1}{\infty}=0$ for the degenerate cases.

\section{Detecting chirality with the determinant.}

In this section, some mostly standard knot theoretic observations are collected in a way to explain and motivate our following investigations.

It is well-known that for any knot $K$ the Alexander polynomial $\Delta_{K}(t)$, normalized so that $\Delta(t)=\Delta(1 / t)$ and $\Delta(1)=1$, satisfies $\Delta_{K}(t)=\Delta_{! K}(t)$. This means that the Alexander polynomial does not distinguish a knot from its mirror image. Its successors (usually) do better. The Jones polynomial satisfies $V_{K}(t)=V_{! K}\left(t^{-1}\right)$. 
The skein polynomial $P[18]$ is a Laurent polynomial in two variables $l$ and $m$ of oriented knots and links and can be defined by being 1 on the unknot and the skein relation

$$
l^{-1} P\left(L_{+}\right)+l P\left(L_{-}\right)=-m P\left(L_{0}\right)
$$

with $L_{ \pm, 0}$ as in (2.2). (This convention uses the variables of [35], but differs from theirs by the interchange of $l$ and $l^{-1}$.) It satisfies $P_{K}(l, m)=$ $P_{! K}\left(l^{-1}, m\right)$. A similar identity holds for Kauffman's polynomial [30]. Such relations mean that achiral knots $K$ (i.e. knots with $K=! K$ ) have selfconjugate polynomials (in one of the variables). In contrast to $\Delta$, this property is not automatic, and so allows for a chirality test. This test is, however, not perfect. The popular example of a chiral knot $K$ (i.e. with $K \neq ! K$ ) but self-conjugate polynomials is $9_{42}$ in the tables of [49, appendix].

Let us recall a paradox appearing argument showing only using $\Delta_{9_{42}}=$ $\Delta_{! 9_{42}}$ that $9_{42} \neq ! 9_{42}$. For this (and also for the purpose of the main work in this paper) we will be concerned with the determinant.

We start first by describing two special cases of the exact property of the determinant of achiral knots, which we will formulate subsequently, because they have occurred in independent contexts and deserve mention in their own right. They allow us to decide about chirality of a knot $K$, at least for $11 / 18$ of the possible values of the determinant.

Proposition 3.1. For any achiral knot $K$, we have $\left|\Delta_{K}(-1)\right| \bmod 36 \in$ $\{1,5,9,13,17,25,29\}$.

Proof. There is an observation (originally likely, at least implicitly, due to Murasugi [41], and applied explicitly in [52]), using the sign of $\Delta(-1)$ (with $\Delta$ normalized as specified). The information of this sign is equivalent to the residue $\sigma \bmod 4$. Whenever $\Delta(-1)<0$, we have $\sigma \equiv 2 \bmod 4$, so in particular $\sigma \neq 0$, and the knot cannot be achiral. This argument applies for example (but not only) for the knot $9_{42}$. Note that the normalization $\Delta(1 / t)=\Delta(t)$ implies $\Delta(1) \equiv \Delta(-1) \bmod 4$, so that if $\Delta(1)=1$, the property $\Delta(-1)<0$ is also equivalent to $\operatorname{det}(K)=|\Delta(-1)| \equiv 3 \bmod 4$ (while $\operatorname{det}(K) \equiv 1 \bmod 4 \Longleftrightarrow \Delta(-1)>0$ ).

Another way to deduce chirality from the determinant is to use the sign of the Lickorish-Millett value $V\left(e^{\pi i / 3}\right)$ [36]. Attention to it was drawn in [60], where it was used to calculate unknotting numbers. Using some of the ideas there, in [54], we observed that this sign implies that if for an achiral knot $3 \mid \Delta(-1)$, then already $9 \mid \Delta(-1)$. Thus, for example, also 
the chirality of $7_{7}$ can be seen already from its Alexander polynomial, as in this case $\Delta(-1)=21$ (although the Murasugi trick does not work here, and indeed $\sigma=0)$.

Combining both criteria, we obtain the claim.

An easy verification shows that all these residues indeed occur.

In view of these opportunities to extract chirality information out of $\Delta$, it appears appropriate to introduce a clear distinction between the terms 'detecting chirality of $K$ ', meant in the sense 'showing that $K$ and $! K$ are not the same knot' (which can be achieved by the above tricks) and 'distinguishing between $K$ and $! K$ ', meant in the sense 'identifying for a given diagram, known a priori to belong to either $K$ or $! K$, to which one of both it belongs' (what they cannot accomplish, but what is the usually imagined situation where some of the other polynomials is not self-conjugate).

The following simple arithmetic consequence is included because of its knot theoretical interpretation, and as it is the starting point of exhibiting some more interesting phenomena described in the next sections.

Recall, that a knot $K$ is rational (or 2-bridge), if it has an embedding with a Morse function having only four critical points (2 maxima and 2 minima). Such knots were classified by Schubert [50], and can be alternatively described by their Conway notation [8]. See for example [1, Section 2.3] for a detailed description. It is well-known that rational knots are alternating (see [5, Proposition 12.14, p. 189]).

Corollary 3.2. Let $p / q$ for $(p, q)=1, p$ odd be expressible as the continued fraction

$$
\left[\left[a_{1}, \ldots, a_{n}, a_{n}, \ldots, a_{1}\right]\right]:=a_{1}+\frac{1}{a_{2}+\frac{1}{\ldots a_{2}+\frac{1}{a_{1}}}}
$$

for a palindromic sequence $\left(a_{1}, \ldots, a_{n}, a_{n}, \ldots, a_{1}\right)$ of even length. Then, $|p| \equiv 1$ or $5 \bmod 12$.

Proof. Observe that $|p|$ is the determinant of the achiral rational knot with Conway notation $\left(a_{1} \ldots a_{n} a_{n} \ldots a_{1}\right)$. The above proposition 3.1 leaves us only with explaining why $9 \nmid p$. The implication $3|\operatorname{det}(K) \Longrightarrow 9| \operatorname{det}(K)$ for $K$ achiral uses $V\left(e^{\pi i / 3}\right)$, counting the number $t_{3}(K)$ of torsion coefficients of the $\mathbb{Z}$-module $H_{1}\left(D_{K}\right)$ divisible by 3 (see [36]). Since self-conjugate polynomials have real values on the complex unit circle, it follows that $t_{3}(K)$ is even. However, for a rational knot $K, H_{1}\left(D_{K}\right)$ is cyclic and non-trivial 
( $D_{K}$ is a lens space), so that there is only one torsion number at all. Thus, $H_{1}\left(D_{K}\right)$ for any achiral rational knot $K$ cannot have any 3 -torsion.

\section{Sums of two squares and determinants of achiral knots.}

\subsection{Realizing sums of two squares as determinants.}

The aim of this section is to establish a partially conjectural correspondence between sums of two squares and the determinant of achiral links. The study of this relation was first initiated in [21], where it was observed that a result of Goeritz [19] implies that the determinant of an achiral knot is the sum of two squares. We shall show here the converse. In fact, we have

Theorem 4.1. An odd natural number $n$ occurs as determinant of an achiral knot $K$ if and only if $n$ is the sum of two squares $a^{2}+b^{2}$. More specifically, $K$ can be chosen to be alternating or prime, and if one can choose $a$ and $b$ to be coprime, then we can even take $K$ to be rational (or 2-bridge).

Note, that we have given an argument different from that of Hartley and Kawauchi for the reverse implication "modulo 36". The congruences in Proposition 3.1 are exactly those which odd sums of two squares leave modulo 36. Clearly, not every number satisfying these congruences is the sum of two squares. The simplest example is 77 .

Additionally to the general case, we also have a complete statement for rational knots.

Theorem 4.2. An odd natural number $n$ is the determinant of an achiral rational knot if and only if it is the sum of the squares of two coprime numbers.

The coprimality condition is clearly restrictive - for example, 49 and 121 are not sums of the squares of two coprime numbers. Moreover, it also implies the congruence modulo 12 proved in corollary 3.2.

Fermat's theorem (see the beginning of Section 2.2) can now be knottheoretically reformulated for example as

Corollary 4.3. If $n=4 x+1$ is a prime, then there is a rational achiral knot with determinant $n$.

Proof. We have $n=a^{2}+b^{2}$ and as $n$ is prime, $a$ and $b$ must be coprime. 
We start by a Proof of Theorem 4.2. For this, recall Krebes's invariant defined in [33]. Any tangle $T$ can be expressed by its coefficients in the Kauffman bracket skein module of the room with four in/outputs (see [57]):

$$
|T|+|=A|+B \bigcup
$$

Definition 4.4. For a tangle $\lcm{T}$, we call $T$ the numerator closure of $T$ and $[T]$ the denominator closure of $T$.

When specializing the bracket variable to $\sqrt{i}(i$ denotes henceforth $\sqrt{-1})$, $A$ and $B$ in (4.1) become scalars.

Then, Krebes's invariant can be defined by

$$
\operatorname{Kr}(T):=\frac{A}{B}=(A, B) \in \mathbb{Z} \times \mathbb{Z} /(p, q) \sim(-p,-q) .
$$

The denominator and numerator of this "fraction" give the determinants of the two closures of $T$. If $T$ is a rational tangle with $\operatorname{Kr}(T)=p / q$ with $p$ odd, then the denominator closure of $T$ is a rational knot denoted by Schubert $[50]$ as $S(p, q)$.

Proof of Theorem 4.2. A rational achiral knot $\left(a_{1} \ldots a_{n} a_{n} \ldots a_{1}\right)$ is of the form

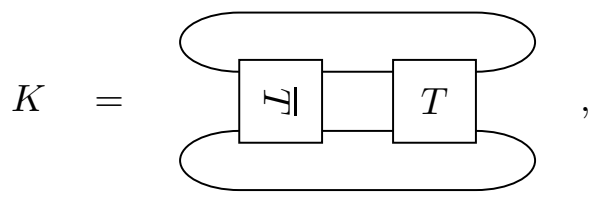

where $T=\left(a_{1} \ldots a_{n}\right)$ is a rational tangle and $\bar{T}$ its mirror image. Because of connectivity reasons, $T$ must be of homotopy type) ( or $\_$, i.e. $\operatorname{Kr}(T)=\left[\left[a_{1}, \ldots, a_{n}\right]\right]=\frac{p}{q}$ with $(p, q)=1$, and exactly one of $p$ and $q$ is odd. Thus, $K$ is the numerator closure of the tangle sum $T+\bar{T}^{T}$, where ${ }^{T}$ denotes transposition. By the calculus introduced by Krebes, his invariant is additive under tangle sum, and invertive under transposition, and so, we have

$$
\frac{\operatorname{det}(K)}{*}=\operatorname{Kr}\left(T+\bar{T}^{T}\right)=\operatorname{Kr}(T)+\frac{1}{\operatorname{Kr}(T)}=\frac{p}{q}+\frac{q}{p}=\frac{p^{2}+q^{2}}{p q} .
$$


To justify our choice of sign in this calculation, that is, that the determinant is $p^{2}+q^{2}$ rather than $p^{2}-q^{2}$, it suffices to keep in mind that the diagram (4.2) is alternating and in calculating the bracket of alternating diagrams no cancellations occur, as explained also in [33]. Thus, we have the 'only if' part.

For the 'if' part, note that if $a$ and $b$ are coprime, then $\frac{a}{b}$ can be expressed by a continued fraction, and hence, as $\operatorname{Kr}(T)$ for some rational tangle $T$. Then, $a^{2}+b^{2}$ (with the above remark on signs) is the determinant of the achiral knot shown in (4.2).

Now, we modify the second part of the proof to deduce Theorem 4.1. In the following, we use Conway's notation for tangle sum and product. (See, for example, again [1, Section 2.3] for a detailed description.)

Proof of Theorem 4.1. Let $n=p^{2}+q^{2}$. If $q=0$, then $K=T(2, p) \# T(2,-p)$ (with $T(2, p)$ denoting the $(2, p)$-torus knot) is an easy example, so let $q \neq 0$. Krebes shows that for any pair $(p, q)$ with at least one of $p$ and $q$ odd, there is a(n arborescent) tangle $T$ with $\operatorname{Kr}(T)=\frac{p}{q}$. In fact, $T$ can be chosen to be the connected sum of a rational tangle and a knot of the type $T(2, p)$ (which can be done in a way the tangle remains alternating). Then, again consider the knot in (4.2) (it is a knot because of the parities of $p$ and $q$ ), and from the proof of Theorem 4.2 one sees that it has the desired determinant $n$.

The knots constructed in (4.2) then are all alternating. It remains to show that they can be made prime (possibly sacrificing alternation). If $(p, q)=1$, then $K$ is rational, and hence prime. Thus, let $n=(p, q)>1$. Then, we can choose $T$ to be of the form

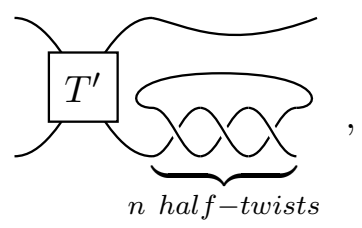

that is, in Conway's notation, $T=(n \cdot \infty) \cdot T^{\prime}$ with a tangle $T^{\prime}$ being a rational tangle $a / b$ with $a=p / n, b=q / n$.

Now, replace the $\infty$-tangle in (4.3) by the "KT-grabber" tangle $K T$ in $[4,17]$. By the same argument as in [17] (or see also $[32,47]$ ), the tangle $T_{1}=$ $(n \cdot K T) \cdot T^{\prime}$ becomes prime, and hence so is then the knot $K_{1}=\overline{T_{1} \cdot T_{1}}$ by proposition 1.3 of [4] (bar denotes tangle closure). As in [4], $K_{1}$ and $K$ have the same Alexander polynomial, so in particular, the same determinant. 
Example 4.5. To demonstrate the elegance of theorem 4.1 as a chirality criterion, we remark that among the prime knots of $\leq 10$ crossings (denoted henceforth according to Rolfsen's tables [49, appendix]) there are 6 chiral knots with self-conjugate HOMFLY polynomial - $9_{42}, 10_{48}, 10_{71}, 10_{91}, 10_{104}$ and $10_{125}$, and this method shows chirality of four of them $-9_{42}, 10_{71}, 10_{104}$ and $10_{125}$, including the two examples $\left(9_{42}\right.$ and $\left.10_{71}\right)$ where additionally, even the Kauffman polynomial is self-conjugate. (For $9_{42}$ and $10_{125}$, the congruence modulo 4 is violated, so that, as remarked on several other places, the signature works as well.)

Remark 4.6. Since slice knots have square determinant, it also follows that if there exists a rational knot $S(p, q)$ which is at the same time achiral and slice, then it will correspond to a Pythagorean triple, that is, be of the Schubert form $S\left(\left(m^{2}+n^{2}\right)^{2}, 2 m n\left(m^{2}-n^{2}\right)\right)$ with $m$ and $n$ coprime.

With regard to theorem 4.1, we conjecture an analogous statement to hold for links.

Conjecture 4.7. An even natural number $n$ occurs as determinant of an achiral link if and only if $n$ is the sum of two squares.

Note that by the above description of (both even or odd) numbers which are sums of two squares, the set of such numbers is closed under multiplication, corresponding to the level of determinants to taking connected sums.

More theoretic results on the square representations (which as said above can also be transcribed knot-theoretically) may be found in [29, 61].

\subsection{Determinants of prime alternating achiral knots.}

Theorem 4.1 naturally suggests the question of how far the properties alternation and primeness can be combined when realizing a sum of two squares as determinant of achiral knots.

In this case, the situation is much more difficult, though. It is easy to see that not every (odd) sum of 2 squares can be realized. The first (and trivial) example is 1 , since the only alternating knot with such determinant is the unknot [9], and it is by definition not prime. However, there are further examples.

Proposition 4.8. Let $n=9$ or $n=49$. Then, there is no prime alternating achiral knot of determinant $n$. 
For its understanding and proof, we recall some standard terminology and results on knot diagrams.

Definition 4.9. The diagram on the left of Figure 1 is called connected sum $A \# B$ of the diagrams $A$ and $B$. If a diagram $D$ can be represented as the connected sum of diagrams $A$ and $B$ such that both $A$ and $B$ have at least one crossing, then $D$ is called disconnected (or composite), else it is called connected (or prime). Equivalently, a diagram is prime if any closed curve intersecting it in exactly two points does not contain a crossing in one of its interior or exterior.

If a diagram $D$ can be written as $D_{1} \# D_{2} \# \cdots \# D_{n}$, and all $D_{i}$ are prime, then they are called the prime (or connected) components/factors of D.

Definition 4.10. The diagram is split, if there is a closed curve not intersecting it, but which contains parts of the diagram in both its interior and exterior.

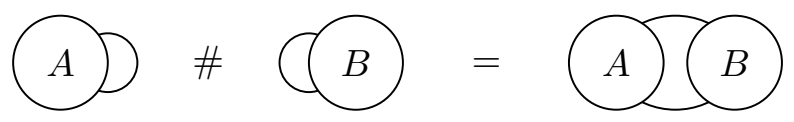

Figure 1.

Definition 4.11. A crossing $q$ in a link diagram $D$ is called nugatory, if there is a closed (smooth) plane curve $\gamma$ intersecting $D$ transversely in $q$ and nowhere else. A diagram is called reduced if it has no nugatory crossings.

Theorem 4.12. ([38]) An alternating reduced diagram of a link $L$ is prime iff $L$ is prime.

Definition 4.13. Let $c(D)$ be the crossing number of a link diagram $D$. Let $c(L)$ be the crossing number of a link $L$, which is the minimal crossing number of all diagrams $D$ of $L$.

By Kauffman-Murasugi-Thistlethwaite, we have

Theorem 4.14. ([31, 43, 58]) Each alternating reduced diagram is of minimal crossing number (for the link it represents). 


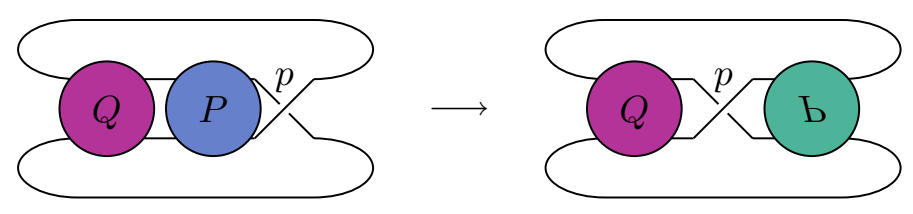

Figure 2: A flype near the crossing $p$

Definition 4.15. A flype is a move on a diagram shown in Figure 2.

By the fundamental work of Menasco-Thistlethwaite, we have

Theorem 4.16. ([39]) For two alternating diagrams of the same prime alternating link, there is a sequence of flypes (and $S^{2}$-moves) taking the one diagram into the other.

In [9], Crowell proved a lower bound on the determinant of alternating links. (A more modern proof can be given for example by the Kauffman bracket.)

Theorem 4.17. (Crowell) If $L$ is a non-split alternating link of crossing number $c(L)=n$, then $\operatorname{det}(L) \geq n$, and if $L$ is not the $(2, n)$-torus link, then $\operatorname{det}(L) \geq 2 n-3$.

By this theorem, one has a bound on the crossing number of an alternating knot of given determinant, so could check for any $n$ in finite time whether it is realized or not. This renders the check for $n=9$ easy. However, the estimate we obtain from Crowell's inequality is intractable in any practical sense for $n=49$. We require an improvement of Crowell's result for achiral alternating links, which relies on his work.

Proposition 4.18. If $L$ is an alternating non-split achiral link of $2 n$ crossings, then $\operatorname{det}(L) \geq n(n-3)$.

Since, we use the checkerboard colorings for the proof, this property holds for the most general notion of achirality for links - we allow the isotopy taking a link to its mirror image to interchange components and/or preserve or reverse their orientations in an arbitrary way. We will define, however, checkerboard colorings later, in Section 6.1, so that we defer the proof of Proposition 4.18 to that later stage.

Remark 4.19. The condition the crossing number of $L$ to be even is no restriction. The crossing number of any alternating achiral (in the most 
general sense, as remarked after Proposition 4.18) link diagram $D$ is even by [39], since flypes preserve the writhe of the alternating diagram, and reversal of any single component alters the writhe by a multiple of 4 . (Any two components have an even number of common crossings by the Jordan curve theorem.) Thus, $w(D) \equiv-w(D) \bmod 4$, and the writhe must be even, and hence so must be the crossing number.

Proof of Proposition 4.8. This is now feasible. Check all the alternating achiral knots in the tables of [23] up to 16 crossings.

There is some possibility that the values of proposition 4.8 are indeed the only exceptions.

Conjecture 4.20. Let $n$ be an odd natural number. Then, $n$ is the determinant of a prime alternating achiral knot if and only if $n$ is the sum of two squares and $n \notin\{1,9,49\}$.

At least this is true up to $n \leq 2000$. A full confirmation of this conjecture is so far not possible, but, we obtain a complete statement for $n$ being a square.

Theorem 4.21. Let $n$ be an odd square. Then, $n$ is the determinant of a prime alternating achiral knot if and only if $n \notin\{1,9,49\}$.

Definition 4.22. Let $\mathcal{L}=\mathbb{Q}\left[t, t^{-1}\right]$ be the Laurent polynomial ring in one variable. For $X, Y \in \mathcal{L}$ write $X \sim Y$ if $X$ and $Y$ differ by a multiplicative unit in $\mathbb{Z}\left[t^{ \pm 1}\right]$, that is, $X(t)= \pm t^{n} Y(t)$ for some $n \in \mathbb{Z}$. For $Y \in \mathcal{L}$ and $a \in \mathbb{Z}$, let $[Y]_{t^{a}}=[Y]_{a}$ be the coefficient of $t^{a}$ in $Y$.

Recall, that a knot $K$ is called strongly achiral, if it admits an embedding into $S^{3}$ pointwise fixed by the (orientation-reversing) involution $(x, y, z) \mapsto(-x,-y,-z)$. Again, depending on the effect of this involution on the orientation of the knot, we distinguish between strongly +achiral and strongly -achiral knots. In [21], properties of the Alexander polynomial of strongly achiral knots are proved.

Theorem 4.23. ([21]) If $K$ is strongly negative amphicheiral, then $\Delta\left(t^{2}\right) \sim$ $F(t) F(-t)$ for some $F \in \mathcal{L}$ with $F(-t) \sim F\left(t^{-1}\right)$. If $K$ is strongly positive amphicheiral, then $\Delta(t)=F(t)^{2}$ for some $F \in \mathcal{L}$ with $F(t) \sim F\left(t^{-1}\right)$. 
For strongly positive amphicheiral knots, Hartley and Kawauchi show more strongly that the Alexander module is a double $A \oplus A$. The same proof applies for strongly positive amphicheiral links, except in the case, when the Alexander module is not (completely) torsion, but then the Alexander polynomial vanishes. Thus, the Alexander polynomial (and in particular, the determinant) is always a square for strongly positive amphicheiral knots and links. This provides some heuristics for the proof of Theorem 4.21. Therefore, although theorem 4.23 is not formally used now, it seems appropriate to mention it here.

Let $S D_{n}$ be the $n$-strand Kauffman diagram algebra given in [30, definition 3.5] with the special parameter $A=\sqrt{i}$ (where $i=\sqrt{-1}$; so that a separate loop trivializes). It can be shown (see [30, Theorem 4.3]) that $S D_{n}$ is linearly generated by the $C_{n}$ loop-free diagrams connecting a pair of $n+n$ points on the bottom and on the top by $n$ lines, where $C_{n}=\frac{1}{n+1}\left(\begin{array}{c}2 n \\ n\end{array}\right)$ is the $n$-th Catalan number. For example, for $n=3$, we have the following 5 elements:

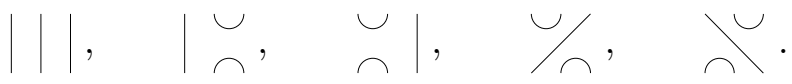

These diagrams also form a basis, as the pairing

$$
\left\langle\frac{1-1}{T_{1}}, \frac{1-1}{T_{2}}\right\rangle_{n}= \begin{cases}1 & \text { if the meander } \\ 0 & \text { else. }\end{cases}
$$

is non-degenerate (see formula (5.18) of [12]). The dimension of $S D_{n}$ is therefore $C_{n}$. The standard multiplicative generators of $S D_{n}$ are

$$
s_{i}=|\cdots| \underset{i i+1}{\smile}|\cdots| \text {. }
$$

The multiplication in $S D_{n}$ is given by stacking up and eventual killing of the resulting diagram if it has a loop. For example, in $S D_{3}$, we have

$$
s_{2}^{2}=\left(\mid \begin{array}{c}
\cup \\
\frown
\end{array}\right)^{2}=0 .
$$

Proof of Theorem 4.21. Since $n$ is a square, it is suggestive by the result of [21] to consider strongly +achiral knots as candidates to realize $n$ as 


\begin{tabular}{|c|c|c|c|c|c|}
\hline & 0 & 0 & 0 & 1 & 1 \\
\hline $\mid$ & 0 & 1 & 0 & 0 & 0 \\
\hline $\begin{array}{l}\cup \\
\frown\end{array}$ & 0 & 0 & 1 & 0 & 0 \\
\hline$/ \cap$ & 1 & 0 & 0 & 0 & 1 \\
\hline & 1 & 0 & 0 & 1 & 0 \\
\hline
\end{tabular}

Table 1.

determinant. We consider diagrams of the type

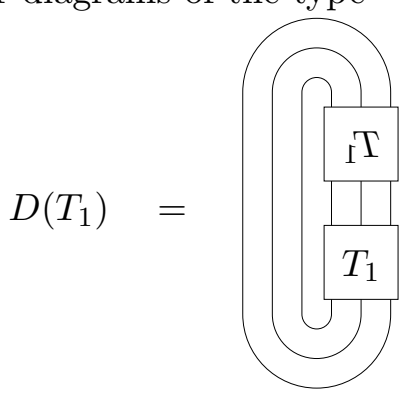

and the pairing $<T_{1}, T_{2}>$ on $S D_{3}(A=\sqrt{i})$ given by Table 1 . We have

$$
<T_{1}, T_{2}>=\left\langle T_{2}, \delta^{-1} T_{2} \delta\right\rangle_{3},
$$

where $\delta=\sigma_{1} \sigma_{2} \sigma_{1}$ is the 3 -strand half-twist, and $\sigma_{i}$ are the usual Artin braid generators.

Then, $\operatorname{det}\left(D\left(T_{1}\right)\right)=<T_{1}, T_{1}>$. Let $T$ be a tangle

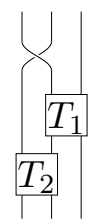

with $T_{1}=A|+| B \smile$ and $T_{2}=X||+Y \smile$. Then,

$$
T=a|||+b| \underset{\frown}{\smile}+c \underset{\frown}{\smile} \mid+d \cup / \curvearrowleft+e
$$


with

$$
\begin{aligned}
a & =X A \\
b=d & =B X \\
c & =A X+B Y+A Y \\
e & =B Y,
\end{aligned}
$$

and

$$
<T, T>=[(X+Y)(A+B)]^{2} .
$$

Whenever $(X, Y)$ and $(A, B)$ are relatively prime, and $X, Y, A, B>0$, one can substitute rational tangles for $T_{1,2}$ obtaining a prime alternating diagram of a strongly +achiral knot. Setting $X=B=1$ and varying $Y$ and $A$, we see that we can cover all cases when $n=p^{2}$ with $p$ composite.

Since we dealt with $p=1$, it remains to consider $p$ prime. If $p \equiv$ $1 \bmod 4$, then (2.6) shows that $n$ has a non-trivial representation as sum of two squares, which then must be coprime. In this case, there is an achiral rational knot of determinant $n$.

Thus, assume $n=p^{2}$ with $p \equiv 3 \bmod 4$ prime. We show now that almost all (not necessarily prime) $p \equiv 3 \bmod 4$ can be realized.

Consider diagrams $D(T)$ for $T$ of the form

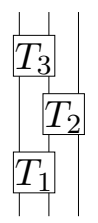

with $T_{1}=X||+Y \longleftarrow, T_{2}=A||+B \smile$ and $T_{3}=C||+D \smile$. We find after multiplying out the polynomial and some manipulation

$$
<T, T>=[X(D A+B C)+Y(B D+A C)]^{2} .
$$

Set $X=1$ and let $Y=k$ vary. The rest is done by choosing small special values for $A, B, C, D$. 


\begin{tabular}{c|c|c|c|c|c|c|c|c|c} 
& $D$ & $B$ & $A$ & $C$ & $D A+B C$ & $B D+A C$ & $\sqrt{<T, T>}$ & $T_{2}$ & $T_{3}$ \\
\hline (1) & 2 & 3 & 2 & 1 & 7 & 8 & $7+8 k$ & $\searrow$ & $\gamma$ \\
(2) & 2 & 7 & 2 & 1 & 11 & 16 & $11+16 k$ & 8 & $\gamma$ \\
(3) & 4 & 3 & 4 & 1 & 19 & 16 & $19+16 k$ & $\gamma$ & 8
\end{tabular}

Examples of the knots thus obtained are given in Figure 3.

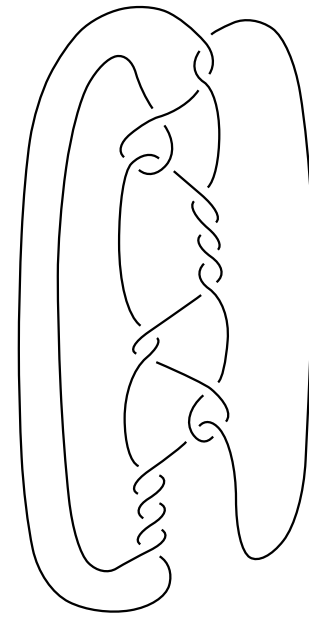

(1), $Y=4$

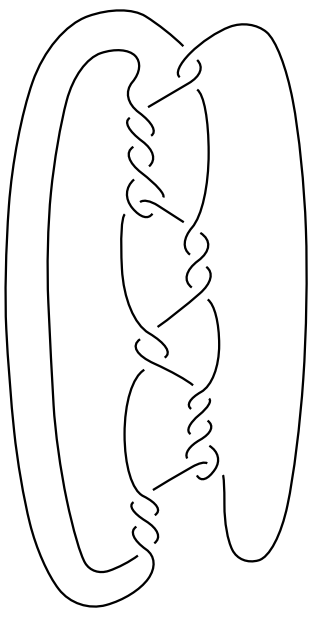

(2), $Y=3$

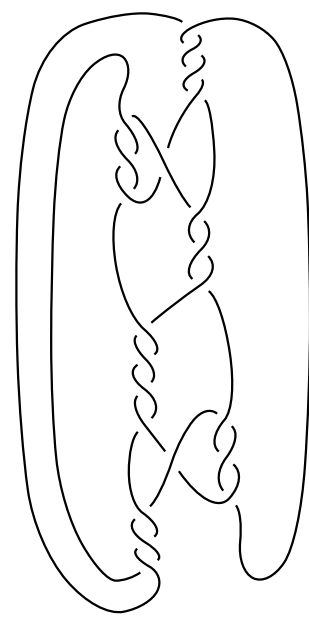

(3), $Y=3$

Figure 3.

All diagrams (and hence knots [38]) are prime for $k \geq 1$. Thus, the only cases remaining to check are for $\sqrt{n}=p \in\{3,7,11,19\}$. For $p=11$, we have $10_{123}$, and for $p=19$, we check the knots in the tables of [23]. We obtain the examples $12_{1019}$ (the closure of the 5 -braid $\left.\left(\sigma_{1} \sigma_{2}^{-1} \sigma_{3} \sigma_{4}^{-1}\right)^{3}\right)$ and $14_{18362}$ (the closure of the 3 -braid $\sigma_{1}^{2} \sigma_{2}^{-3} \sigma_{1}^{2} \sigma_{2}^{-2} \sigma_{1}^{3} \sigma_{2}^{-2}$ ). The cases $p=3,7$ were dealt with in Proposition 4.8.

Remark 4.24. We showed that in fact, we can realize any $n$ stated in the theorem by a strongly +achiral or rational knot, so, in particular, by a strongly achiral knot, since an achiral rational knot is known to be strongly 
-achiral (see [21]). It may be possible to eliminate the need of rational knots when allowing the further exception $n=25$.

To examine the general case of $n$ (not only perfect squares), one needs to consider larger series of examples. Because of Theorem 4.23, the knots should not (only) be strongly +achiral. We just briefly discuss one series to explain some of the occurring difficulties.

A natural way to modify the examples in the proof of theorem 4.21 to be -achiral is to consider (braid type) closures of tangles like

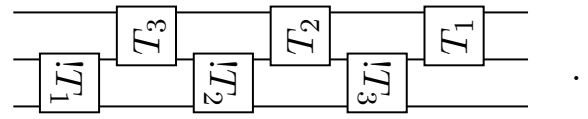

By choosing arborescent alternating tangle diagrams $T_{1,2,3}$, this pattern gives rise to a series of achiral alternating knots. However, we have

Claim 4.25. Infinitely many $n$, which are sums of two squares, are not realized as determinants of the alternating closure of (4.5) for arborescent alternating diagram tangles $T_{1,2,3}$.

Remark 4.26. Note that an alternating (and) arborescent tangle may not have an alternating (and) arborescent diagram. Take, for example, the 3braids $\sigma_{1}^{3} \sigma_{2}^{2} \sigma_{1}^{-2} \sigma_{2}^{-3}$ or $\sigma_{1}^{3} \sigma_{2}^{3} \sigma_{1}^{-3} \sigma_{2}^{-3}$, and close up one strand. The braids can be turned into alternating words whose closure gives the alternating diagrams of the knots $8_{17}$ and $10_{99}$, and these alternating diagrams are not arborescent.

Proof. Using the Kauffman bracket skein module coefficients

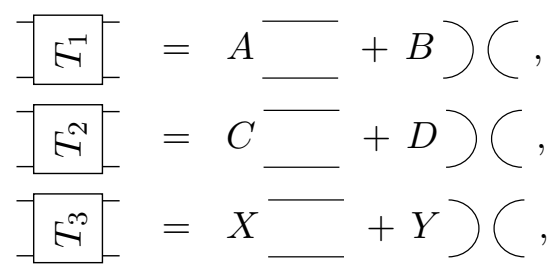

one finds, as before, an expression for $\operatorname{det}(K)$ as polynomial in $A, B, C, D, X, Y$ and after some manipulation, arrives at $\operatorname{det}(K)=f_{1}^{2}+f_{2}^{2}$ with

$$
\begin{gathered}
f_{1}(A, B, C, D, X, Y)=X(A D+B C)+Y(A C+B D) \quad \text { and } \\
f_{2}(A, B, C, D, X, Y)=Y(A D-B C) .
\end{gathered}
$$


The correctness of the square decomposition is straightforward to check, but for finding it, it is helpful to notice that the substitutions $Y=0$ and $A=C, B=D$ turn the knots into strongly +achiral ones. Since we know that such knots have square determinant, it is suggestive that each of the above substitutions makes one of $f_{1}$ or $f_{2}$ vanish.

Consider a number of the form $n=5 p^{2}$ with $p \equiv 3 \bmod 4$ prime. Assume, $n$ can be written as $f_{1}^{2}+f_{2}^{2}$ with $f_{1,2}$ as in (4.6) for $A, B, C, D, X, Y \in$ $\mathbb{N}$. Then, $\left\{f_{1}, f_{2}\right\}=\{p, 2 p\}$, since obviously $f_{1}, f_{2} \geq 0$, and for so restricted $f_{1,2}$, the representation $n=f_{1}^{2}+f_{2}^{2}$ is unique up to interchange. Starting with the product form of $f_{2}$, and making a slightly lengthy, but straightforward calculation in cases, one then finds that $\{A, B\},\{C, D\}$ or $\{X, Y\}$ is one of $\{0, p\},\{p, p\}$, or $\{p, 2 p\}$. However, it is an easy inductive exercise using Krebes calculus to prove that no alternating arborescent diagram tangle $T_{i}$ has such pair of Kauffman bracket skein module coefficients. Therefore, the above series cannot realize determinants of this form.

Of course, the template (4.5) is not exhaustive, and indeed, on the other hand, the small cases of the form $n=5 p^{2}$ (for $p \leq 11$ ) are realized by knots with alternating diagrams of Conway polyhedron $8^{*}$.

Then, one can consider more patterns and write down more complicated polynomials, every time wondering whether each (at least sufficiently large) number is realized by (at least some of) these polynomials. Presently, such problems in number theory seem very difficult. (One classic example is the determination of the numbers $G(n)$ and $g(n)$ in Waring's problem, see for example $[11,22,20]$.) Therefore, Conjecture 4.20 may be hard to (confirmatively) approach as of now.

\subsection{Determinants of unknotting number one achiral knots.}

We conclude our results on sums of two squares by a related, although somewhat auxiliary, consequence of the unknotting number theorem of Lickorish [34] and its refined version given in [54].

Let $u_{ \pm}$denote the signed unknotting number, the minimal number of switches of crossings of a given sign (see (2.2)) to a crossing of the reversed sign needed to unknot a knot, or infinity if such an unknotting procedure is not available. (This is somewhat different from the definition of [6].)

Thus, a knot $K$ has $u_{+}(K)=1$ (resp. $u_{-}(K)=1$ ) if it can be unknotted by switching a positive (resp. negative) crossing in some of its diagrams. 
Proposition 4.27. Let $K$ be a knot with $u_{+}=u_{-}=1$ (for example, an achiral unknotting number one knot). Then, $\operatorname{det}(K)$ is the sum of two squares of coprime numbers.

Proof. Clearly, $\sigma(K)=0$, so that any of the relevant crossing changes do not alter the signature. Now, consider the linking form $\lambda$ on $H_{1}\left(D_{K}\right)([34])$. For $g, h \in H_{1}\left(D_{K}\right)$ and $n=\operatorname{det}(K)$, the pairing $\lambda(g, h) \in \mathbb{Q} / \mathbb{Z}$ is defined as $1 / n$ times the algebraic intersection number of $g$ with a surface bounding $n \cdot h$ (which is homologically trivial). By the refinement of Lickorish's theorem given in [54], the properties $u_{+}=u_{-}=1$ imply $\lambda\left(g_{ \pm}, g_{ \pm}\right)= \pm 2 / n \in \mathbb{Q} / \mathbb{Z}$ for some generators $g_{ \pm}$of $H_{1}\left(D_{K}\right)$. Thus, $2 l^{2}=-2 h^{2}$ for some $l, h \in \mathbb{Z}_{n}^{*}$. The structure of the group $\mathbb{Z}_{n}^{*}$ of units in $\mathbb{Z}_{n}=\mathbb{Z} / n \mathbb{Z}$ is known; see e.g. [ 63 , Exercise 1, Section 5, p. 41]. From this structure and (2.10), we see that the number of square roots of -1 in $\mathbb{Z}_{n}^{*}$ (for $n>1$ odd) is identical to $r_{2}^{0}(n)$. (D. Zagier remarked to me that one can in fact give a natural bijection between the square roots of -1 in $\mathbb{Z}_{n}^{*}$ and representations of $n$ as sum of coprime squares.) Now, when $2 l^{2}=-2 h^{2}$, then $\mathbb{Z}_{n}^{*}$ possesses square roots of -1 .

There are also several questions opened by proposition 4.27. Setting

$$
S:=\left\{a^{2}+b^{2}:(a, b)=1,2 \nmid a+b\right\},
$$

we have the inclusions

$$
\begin{aligned}
S_{a}:=\{\operatorname{det}(K): K \text { achiral, } u(K)=1\} \\
\quad \subset S_{ \pm}:=\left\{\operatorname{det}(K): u_{+}(K)=u_{-}(K)=1\right\} \subset S .
\end{aligned}
$$

It is a natural question whether (and which of) these inclusions are proper.

This seems much more difficult to decide than the proof of Theorems 4.1 and 4.2. There is no such straightforward procedure available to exhaust all values in $S$, and to show a proper inclusion, one will face the major problem of deciding about unknotting number one.

After a computer experiment with the knot tables and tools available to me, the smallest $x \in S$ I could not decide to belong to $S_{ \pm}$is 349 , whereas the smallest possible $x \in S$ with $x \notin S_{a}$ is only 17 . Contrary to Theorems 4.1 and 4.2 , very many entries have been completed only by non-alternating knots (which have smaller determinant than the alternating ones of the same crossing number), and in fact, we can use the number 17 to show that for this problem non-alternating knots definitely need to be considered. 
Example 4.28. From theorem 4.17, we see that except for the $(2,17)$-torus knot, any alternating knot of determinant 17 has $\leq 10$ crossings. A direct check shows that the only such knots of $\sigma=0$ are $8_{3}$ and $10_{1}$. However, $u\left(8_{3}\right)=2$ as shown by Kanenobu-Murakami [28], and that $10_{1}$ cannot simultaneously have $u_{+}=u_{-}=1$ follows by refining their method (see [53]). Thus, there is no alternating knot of determinant 17 with $u_{+}=u_{-}=1$, and the inclusion

$$
S_{a \pm}:=\left\{\operatorname{det}(K): K \text { alternating, } u_{+}(K)=u_{-}(K)=1\right\} \subset S
$$

is proper. (Contrarily, there is a simple non-alternating knot, $9_{44}$, with $u_{+}=$ $u_{-}=1$ and determinant 17.) Is it infinitely proper, i.e., is $\left|S \backslash S_{a \pm}\right|=\infty$ ?

It is suggestive that considering the even more restricted class of rational knots, the inclusions are infinitely proper. We confirm this for achiral unknotting number one rational knots.

Proposition 4.29. $S \backslash\{\operatorname{det}(K): K$ achiral and rational, $u(K)=1\}$ is infinite. (In fact, this set contains infinitely many primes.)

Proof. These knots were classified in [55, Corollary 2.2] to be those with Conway notation $(n 11 n)$ and $\left(3(12)^{n} 1^{4}(21)^{n} 3\right)$. It is easy to see therefore that the inclusion

$$
\{\operatorname{det}(K): K \text { achiral and rational, } u(K)=1\} \subset S
$$

is infinitely proper. For example, the determinant of both series grows quadratically resp. exponentially in $n$, so that

$$
\sum_{K=S(p, q)} \frac{1}{p}<\infty
$$

achiral, $u(K)=1$

while $\sum_{p \in S} \frac{1}{p}=\infty$. (Already, by Dirichlet $\sum_{\substack{p \equiv 1(4) \\ \text { prime }}} \frac{1}{p}=\infty$, see $[63$,

Korollar, p. 46].)

It appears feasible to push the method of [55] further to show the same also for rational knots with $u_{+}=u_{-}=1$ (although I have not carried out a 
proof in detail). By refining Kanenobu-Murakami, we first show that there is a crossing of the same sign unknotting the alternating diagram of the rational knot (see [53]). Then, consider alternating rational knot diagrams with two unknotting crossings (it does not even seem necessary to have them any more of different sign), and apply the same argument as above, using [55, corollary 2.3] and the remark after its proof.

\section{Enumeration of rational knots by determinant.}

The results of Section 3 can be used to enumerate rational knots by determinant. We have for example:

Proposition 5.1. The number $c_{n}$ of achiral rational knots of given determinant $n$ is given by

$$
c_{n}= \begin{cases}1 / 2 r_{2}^{0}(n) & \text { if } n>2 \text { odd } \\ 0 & \text { else }\end{cases}
$$

Proof. Use the fact that there is a bijective correspondence between the rational tangle $T$ in a diagram (4.2) of an achiral rational knot $K$ and its Krebes invariant $p / q$ (with $p \geq q$ and $(p, q)=1$ ) giving $\operatorname{det}(K)=p^{2}+q^{2}$.

From (2.10), we then obtain

Corollary 5.2. The number of achiral rational knots of given determinant $n$ is either zero or a power of two.

As a practical application of the argument proving Proposition 5.1, we can consider the achiral rational knots $(1 \ldots 1)$ and $(31 \ldots 13)$ (with the sequence of numbers having even length) and the tangles $T$ obtained from the halves of the palindromic sequence. This way, one arrives to a knot theoretical explanation of the identities

$$
F_{2 n+1}=F_{n}^{2}+F_{n+1}^{2} \quad \text { and } \quad L_{2 n+1}+2 L_{2 n}=L_{n}^{2}+L_{n+1}^{2},
$$

where $F_{n}$ is the $n$-th Fibonacci number $\left(F_{1}=1, F_{2}=1, F_{n}=F_{n-1}+F_{n-2}\right)$ and $L_{n}$ is the $n$-th Lucas number $\left(L_{1}=2, L_{2}=1, L_{n}=L_{n-1}+L_{n-2}\right)$. Thus, we have the property ${ }^{2}$

\footnotetext{
${ }^{2}$ which for $F_{2 n+1}$ I remember from an old issue of the Bulgarian journal "Matematika" and recently found (by electronic search) conjectured in [59] and proved in $[62]$
} 
Proposition 5.3. There are achiral rational knots with determinant $F_{2 n+1}$ and $L_{2 n+1}+2 L_{2 n}$ for any $n$, or equivalently, any (prime) number $4 x+3$ does not divide $F_{2 n+1}$ and $L_{2 n+1}+2 L_{2 n}$.

A similar enumeration can be done for arbitrary rational knots of given (odd) determinant $n$, and one obtains

Proposition 5.4. The number of rational knots of determinant $n(n>1$ odd), counting chiral pairs once, is

$$
\frac{1}{4}\left\{\phi(n)+r_{2}^{0}(n)+2^{\omega(n)}\right\}
$$

with $r_{2}^{0}(n)$ being as in (2.11), $\omega(n)$ denoting the number of different prime divisors of $n$ and $\phi(n)=\left|\mathbb{Z}_{n}^{*}\right|$ being Euler's totient function. Counting chiral pairs twice one has the somewhat simpler expression

$$
\frac{1}{2}\left\{\phi(n)+2^{\omega(n)}\right\} .
$$

Proof. Consider the first formula. We apply Burnside's lemma on the action of $\mathbb{Z}_{2} \times \mathbb{Z}_{2}$ on $\mathbb{Z}_{n}^{*}$ given by additive inversion in the first component and multiplicative inversion in the second one. In (5.2), the second and third terms in the braced expression come from counting the square roots of $\mp 1$ in $\mathbb{Z}_{n}^{*}$. These numbers follow from the structure of this group $\mathbb{Z}_{n}^{*}$, as remarked in the proof of Proposition 4.27. The second formula is obtained similarly and more easily.

Remark 5.5. The functions $\omega(n)$ and $\phi(n)$ are hard to calculate for sufficiently large numbers $n$ by virtue of requiring the prime factorization of $n$, but the expression in terms of these classical number theoretical functions should be at least of theoretical interest.

In a similar way, one could attempt the enumeration by $u_{p}$ of unknotting number one rational knots of determinant $p$ using [28], seeking again an expression in terms of classical number theoretical functions. Obviously from the result of Kanenobu-Murakami, we have

$$
u_{p} \leq 2^{\omega((p+1) / 2)-1}+2^{\omega((p-1) / 2)-1}-1,
$$

with the powers of two counting the representations of $(p \pm 1) / 2$ as the product of two coprime numbers $n_{ \pm}$and $m_{ \pm}$up to interchange of factors 
and the final ' -1 ' accounting for the double representation of the twist knot for $m_{+}=m_{-}=1$. However, to obtain an exact formula, one encounters the problem that, beside the twist knot, some other knot may arise from different representations (although this does not occur often and the inequality above is very often sharp). For example, for $p=985$ the knot $S(985,288)=$ $S(985,697)$ occurs for the representations $m_{+}=29$ and $m_{-}=12$. D. Zagier informed me that he has obtained a complete description of the duplications of the Kanenobu-Murakami forms when considering $q$ in $S(p, q)$ only up to additive inversion in $\mathbb{Z}_{p}^{*}$. According to him, however, considering the (more relevant) multiplicative inversion renders the picture too complicated and number theoretically unilluminating.

\section{Spanning trees in self-dual graphs.}

\subsection{Spanning trees in planar graphs and checkerboard colorings.}

Here, we discuss an interpretation of our results of Section 4 in graph theoretic terms.

Theorem 6.1. Let $n$ be an odd natural number. Then, $n$ is the number of spanning trees in a planar self-dual graph if and only if $n$ is the sum of two squares.

The proof of this theorem relies on a construction that links graph and knot theory, by associating to an alternating knot (or link) diagram $D$ its checkerboard graph (see e.g. [30]).

The checkerboard coloring of a link diagram is a map

$$
\{\text { regions of } D\} \rightarrow\{\text { black, white }\}
$$

s.t. regions sharing an edge are always mapped to different colors. (A region is called a connected component of the complement of the plane curve of $D$, and an edge a part of the plane curve of the diagram between two crossings.)

The checkerboard graph of $D$ is defined to have vertices corresponding to black regions in the checkerboard coloring of $D$, and an edge for each crossing $p$ of $D$ connecting the two black regions opposite at crossing $p$ (so multiple edges between two vertices are allowed).

This construction defines a bijection

\{alternating diagrams up to mirroring $\} \longleftrightarrow\{$ planar graphs up to duality $\}$ 
Duality of the planar graph corresponds to switching colors in the checkerboard coloring and has the effect of mirroring the alternating diagram if we fix the sign of the crossings so that each crossing looks like

rather than (Note that duality essentially depends on the planar embedding of the graph.)

Definition 6.2. A graph is 2-connected if the removal of any $\leq 2$ edges does not disconnect it. A loop (edge) is an edge connecting with both ends the same vertex. A cut vertex is a vertex, which is the endpoint of a loop edge, or which disconnects the graph, when removed together with all its incident edges.

Then, we have the following useful observation:

Lemma 6.3. Let $D$ be an alternating diagram and $G$ its checkerboard graph. Then, $D$ is prime iff $G$ has no cut vertex. $D$ is reduced iff $G$ has no loop edges and is 2-connected.

Note that, in our terminology, reducible diagrams are always composite. (Equivalently, graphs with loop edges, or such that are not 2-connected, always have a cut vertex.)

Lemma 6.4. $\operatorname{det}(D)$ equals the number $t(G)$ of spanning trees in a checkerboard graph $G$ of $D$ for any alternating link diagram $D$.

Proof. By the Kauffman bracket definition of the Jones polynomial $V$, for an alternating diagram $D$, the determinant $\operatorname{det}(D)=\left|\Delta_{D}(-1)\right|=\left|V_{D}(-1)\right|$ can be calculated as follows (see [33]).

Consider $\hat{D} \subset \mathbb{R}^{2}$, the (image of) the associated immersed plane curve(s). For each crossing (self-intersection) of $\hat{D}$, there are 2 ways to splice it:

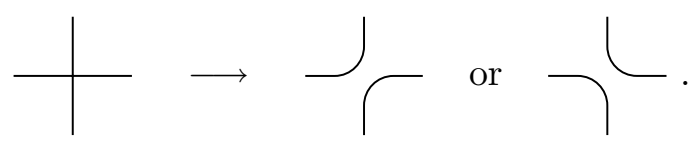

We recall that a choice of splicing for each crossing is called a state. Then, $\operatorname{det}(D)$ is equal to the number of states $S$, so that the resulting collection of disjoint circles has only one component (a single circle), i.e. $|S|=1$ in (2.3). We call such states $S$ monocyclic. 
Let $\Gamma$ be a spanning tree of the checkerboard graph $G$ of $D$. Define a state $S(\Gamma)$ as follows: for any edge $v$ in $G$, set

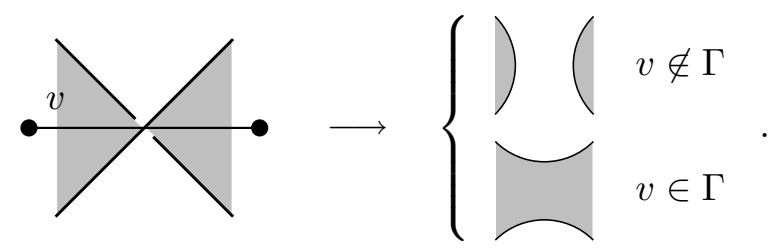

Then, $S$ gives a bijection between monocyclic states of $D$ and spanning trees of $G$.

The following relationship is easy and useful.

Proposition 6.5. $\operatorname{det}(L)$ is odd if and only if $L$ is a knot.

Proof. Since $\operatorname{det}(L)=\left|\Delta_{L}(-1)\right|$ and it is known that for an $n$-component link $L,\left(t^{1 / 2}-t^{-1 / 2}\right)^{n-1} \mid \Delta_{L}(t)$, we have that $2^{n-1} \mid \operatorname{det}(L)$. Thus, $\operatorname{det}(L)$ is odd only if $L$ is a knot. The converse is also true, since for a knot $K$, we have $\Delta_{K}(1)=1$, and $\Delta_{K}(-1) \equiv \Delta_{K}(1) \bmod 2$.

Proposition 6.6. Any odd number $n$ is the number of spanning trees in a planar self-dual graph without cut vertex if and only if $n$ is the determinant of a prime alternating diagram of an achiral knot.

Proof. That the first side of the equivalence implies the second side is clear from Proposition 6.5 and Lemma 6.3. For the reverse direction, let $K$ be a prime amphicheiral knot with reduced alternating diagram $D$ and checkerboard graph $G$ with $n$ spanning trees. ( $G$ no cut vertex.) By the result of [10], there exists a knot $K^{\prime}$ with alternating diagram $D^{\prime}$, whose checkerboard graph $G^{\prime}$ is isomorphic to $G$ (i.e. differs from $G$ only by the planar embedding), and $G^{\prime}$ is self-dual. Since cut vertices do not depend on the planar embedding, $G^{\prime}$ still does not have a cut vertex, and has $n$ spanning trees.

Proof of Theorem 6.1. If $n$ is the number of spanning trees of a planar self-dual graph $G$, then its associated alternating diagram $D$ is isotopic by $S^{2}$-moves to its mirror image. Since $n$ is assumed odd, $D$ is a knot diagram. 
Thus, the number of spanning trees of $G$, which by Lemma 6.4 is equal to $\operatorname{det}(D)$, is of the form $p^{2}+q^{2}$ by Theorem 4.1.

Contrarily, assume that $n=p^{2}+q^{2}$. Take the checkerboard graph $G$ of the diagram in (4.2) constructed in the proof of Theorem 4.2 with $T$ of the form (4.3). This diagram has the property of being isotopic to its mirror image by $S^{2}$-moves only (and no flypes), so that its (self-dual) checkerboard graph $G$ is the one we sought.

Using checkerboard colorings, we will now give an argument for proposition 4.18. It will be useful to have the following terminology set up and easy observation pointed out.

Definition 6.7. Let $D_{1,2}$ be alternating diagrams and $G_{1,2}$ their checkerboard graphs. The checkerboard graph of the connected sum $D_{1} \# D_{2}$ is called the join (or block sum) of $G_{1,2}$. (See also [44].)

Note that, in the same way as performing the connected sum depends on the choice of particular segments of the diagrams, the join depends on the choice of particular vertices of graphs.

Lemma 6.8. The determinant is multiplicative under connected sum of diagrams. The number of spanning trees is multiplicative under join of graphs (no matter in which way these operations are performed).

Proof of Proposition 4.18. First, assume $L$ is prime. Consider the (planar) checkerboard graph $G$ of an alternating diagram $D$ of $L$. White regions of the checkerboard coloring of $D$ correspond (per convention) to faces of $G$, and black regions to vertices of $G$, and by Lemma $6.3, G$ has no cut vertex. Then, use Theorem 2.4 of [9]. Since $D$ has $n+1$ black and white regions, we have $t(G)=\operatorname{det}(L) \geq n^{2}+1$.

Now, assume $D$ is alternating and composite (but reduced). All prime factors $D_{1}, \ldots, D_{l}$ of $D$ are (reduced and) alternating. By [31, 43, 58], we have $\sum c\left(D_{i}\right)=c(D)=c(L)=2 n$. By the uniqueness of prime factor decomposition, we can assume that for $i=1,3,5, \ldots, 2 k-1<l$ the diagrams $D_{i}$ and $D_{i+1}$ are (as unoriented diagrams) mirror images of each other, and that the $D_{j}$ for $j=2 k+1, \ldots, l$ are transformable (as unoriented diagrams) by flypes into their own mirror images.

Let $G_{j}$ be the checkerboard graphs of $D_{j}$ and $G_{j}$ have $2 q_{j}$ edges. Then we know from Crowell's inequality that $\operatorname{det}\left(D_{j}\right)=t\left(G_{j}\right) \geq q_{j}^{2}+1$. Let $p_{i} \geq 2$ be the number of crossings of $D_{i}$. Then by Crowell's inequality for 
general alternating diagrams (Theorem 4.17) and Lemma 6.8, we obtain $\operatorname{det}\left(D_{i} \# D_{i+1}\right) \geq p_{i}^{2}$. Thus,

$$
\operatorname{det}(L) \geq \prod_{i=1}^{k} p_{2 i-1}^{2} \cdot \prod_{j=2 k+1}^{l}\left(q_{j}^{2}+1\right),
$$

where $\sum_{i=1}^{k} p_{2 i-1}+\sum_{j=2 k+1}^{l} q_{j}=n$ and $p_{i} \geq 2$.

It is easy to prove that the minimal value of the r.h.s. of (6.1) is not smaller than $n^{2}-n+2$.

Use induction on $n$. The cases $n=1,2$ and $l-k=1$ are easy. Otherwise, choose $p_{i}, q_{j}$. First, assume some $p_{i}$ exist (i.e. $k>0$ ). Since $l-k>1$, we have $p:=p_{1}<n$. If $p=n-1$, then $l=3$ and $k=q_{3}=1$, and we are led to the inequality $n^{2}-n+2 \leq 2(n-1)^{2}$, which is true for $n \geq 3$. Then, it suffices to show

$$
\left(n-\frac{1}{2}\right)^{2}+\frac{7}{4} \leq p^{2}\left[\left(n-p-\frac{1}{2}\right)^{2}+\frac{7}{4}\right],
$$

with $2 \leq p \leq n-2$.

Postpone this task for a moment, and turn to the other case. That is, assume no $p_{i}$ exists $(k=0)$. Order the $q_{j}$ decreasingly. Since $l=l-k>1$, there are at least two $q_{j}$, and we have $q:=q_{1}<n$. If $q=n-1$, then $l=2$ and $q_{2}=1$. This leads to $n^{2}-n+2 \leq 2(n-1)^{2}+2$, which is true for $n \geq 3$. If $q=1$, then $l=n$ and all $q_{j}=1$, and we are led to prove $n^{2}-n+2 \leq 2^{n}$, which is likewise true for $n \geq 3$. So, assume we have $2 \leq q \leq n-2$. Then, inductively it is to show

$$
\left(n-\frac{1}{2}\right)^{2}+\frac{7}{4} \leq\left(q^{2}+1\right)\left[\left(n-q-\frac{1}{2}\right)^{2}+\frac{7}{4}\right] .
$$

The inequality (6.2) implies (6.3). Thus, consider only (6.2). Assume $n \geq 5$, since for $n=3$, we excluded all possible $p$, and for $n=4$, the only value in question for $p$ is 2 , for which (6.2) is directly checked. Removing in (6.2) the terms $7 / 4$, we can simplify and strengthen it to

$$
n-\frac{1}{2} \leq\left(n-p-\frac{1}{2}\right) \cdot p
$$

The r.h.s. of (6.4) is minimal, when $p$ is as close as possible to 0 or $n-\frac{1}{2}$, which happens for $p=n-2$. For this $p$, the inequality (6.4) holds (and hence (6.2) also does) if $n \geq 5$. 
Remark 6.9. Proposition 4.18 was given in a weaker form than we prove it, because a previous (and independent from Crowell) proof of this weaker form was found by myself. Note also that for Proposition 4.8, it suffices to consider prime diagrams $D$, for which to prove the inequality $\operatorname{det}(D) \geq n^{2}+1$, only the initial reference to Crowell suffices. However, Proposition 4.18 was later used and referred to in this stated form in Theorem 3, Part 14 of [44]. Thus, the formulation and the additional argument in the proof are (now inconvenient, but) appropriate.

It is useful to remark that we can now amplify the inequality deduced in [44].

Corollary 6.10. For any planar self-dual 2-connected loop-free (not necessarily even valence) graph $G$ with $n$ edges, we have $t(G) \geq 1 / 4 n^{2}-n / 2+2$, and if $G$ has (additionally) no cut vertex, then $t(G) \geq 1 / 4 n^{2}+1$.

One can also reformulate Theorem 4.21 and Conjecture 4.20 graphtheoretically, using Proposition 6.6.

Theorem 6.11. Let $n$ be an odd perfect square. Then, $n$ is the number of spanning trees in a planar self-dual graph without cut vertex if and only if $n \neq 9,49$.

Note that the case $n=1$ corresponds to the unknot. While according to our Definition 4.9, its 0-crossing diagram is prime (and its checkerboard graph, which is a single isolated vertex, is self-dual and without cut vertex), the unknot is said not to be prime by convention.

Conjecture 6.12. Let $n$ be an odd natural number. Then, $n$ is the number of spanning trees in a planar self-dual graph without cut vertex if and only if $n$ is the sum of two squares and $n \neq 9,49$.

\subsection{Strongly +achiral knots and squares in recurrent sequences.}

Consider the wheel graph $W_{k}$ of $k+1$ vertices.

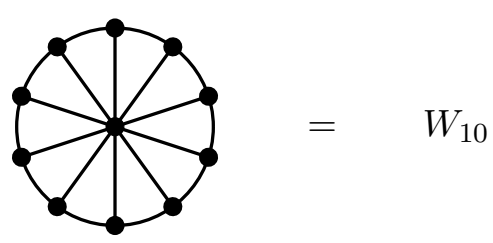


The number $c_{k}$ of spanning trees in $W_{k}$ can be computed by distinguishing the number of edges of the spanning tree incident to the central vertex of the wheel, and counting the spanning forests of the necklace graph remaining from the spanning tree in $W_{k}$ after removing the central vertex. This was carried out in [45, p. 469-470]. The resulting sequence is $1,5,16,45,121, \ldots$ and can be expressed by the Fibonacci and Lucas numbers given before Proposition 5.3 as

$$
c_{k}=L_{2 k}-2=F_{2 k}+2 \sum_{i=1}^{k-1} F_{2 i} .
$$

(The equivalence of both expressions can be shown by elementary generating series arguments.) Another occurrence of this sequence is in [48] as the number of certain unimodular matrices. See also [46] and [51, sequence 004146].

A closer look on the numbers $c_{k}$ reveals that for odd $k, c_{k}$ is a (perfect) square. Although there have been, in particular recently, many related results, e. g. [7, 13, 14, 15, 16, 37, 40], I did not find an explicit statement of this observation. Nonetheless, it is suggestive that this phenomenon should not be the result of an accidental coincidence, and indeed a combinatorial explanation of it is possible by writing down the explicit formula for $L_{k}$

$$
L_{k}=\left(\frac{1+\sqrt{5}}{2}\right)^{k-1}+\left(\frac{1-\sqrt{5}}{2}\right)^{k-1} .
$$

However, the same phenomenon occurs also with (the odd index members of) some closely related sequences like

$$
c_{k}^{\prime}=c_{k}+F_{k}^{2}+2 F_{2 k} \quad \text { and } \quad c_{k}^{\prime \prime}=c_{k}+4 F_{k}^{2}+4 F_{2 k} .
$$

We will give here an explanation of such a phenomenon in terms of knot theory (showing how to find further such sequences and prove their squareness in a much easier and more elegant way than via the naive arithmetical approach). It turns out, that the numbers $c_{k}$ occur as determinants of some strongly +achiral (alternating 3-braid) knots and links.

Lemma 6.13. $\operatorname{det}\left(\left(\widehat{\sigma_{1} \sigma_{2}^{-1}}\right)^{k}\right)=c_{k}$ (where hat denotes braid closure).

Proof. Using the wheel graph description of $c_{k}$, the claim becomes a special case of Lemma 6.4. 
The links of the form $\left(\widehat{\sigma_{1} \sigma_{2}^{-1}}\right)^{k}$ have been considered for a while, for example in [26] (at least in the knot case $3 \nmid k$ ). There it was observed that for odd $k$ (for which the knots are also called "turks head knots"), the braid $\left(\widehat{\sigma_{1} \sigma_{2}^{-1}}\right)^{k}$ is of the form $\beta \bar{\beta}$, where $\bar{\beta}$ is obtained from $\beta \in B_{n}$ by the map $\sigma_{i}^{ \pm 1} \mapsto \sigma_{n-i}^{\mp 1}$. Hence, $\left(\widehat{\sigma_{1} \sigma_{2}^{-1}}\right)^{k}$ is strongly +achiral. This, together with Lemma 6.13 and Theorem 4.23 shows

Proposition 6.14. $c_{k}$ is a square number for $k$ odd. Hence, so is the number of spanning trees in wheel graphs with an odd number of spokes or the by 2 decreased Lucas number $L_{k}$ with $k \equiv 2 \bmod 4$.

The fact that the odd index number knots are still at least achiral (in the usual sense), shows that $c_{k}$ for $k$ even is at least the sum of two squares. Unfortunately, contrary to the result obtained for the odd index parity, there seems no knot theory tool available to examine effectively the even index number case. However, the test of the prime decomposition of $c_{k}$ leads to conjecture even more, namely that these numbers are of the form $c_{k}=5 a_{k}^{2}$ for $k$ even, and this can be indeed confirmed from the explicit formula (6.6) for $L_{k}$. (This observation seems to fit into a more general pattern conjecturally described at the end of this subsection.) The knottheoretic counterpart of the non-squareness of $c_{k}$ for even $k$ is true also by different arguments. It was remarked in [56] how the work of Murasugi [42] on the Alexander polynomial of periodic knots implies that the Alexander polynomial of any non-trivial knot (and analogously, link), which is the closure of the square of some braid (here $\left(\sigma_{1} \sigma_{2}^{-1}\right)^{k / 2}$ ), is not a square, so that the knot is not strongly +achiral (although in our situation, it is ordinarily +achiral).

On the other hand, for odd $k$, it is clear that now a similar procedure can be applied to more general braids. For example, applying the argument to $\beta \bar{\beta}$ with $\beta=\sigma_{1}\left(\sigma_{1} \sigma_{2}^{-1}\right)^{k}$ and $\beta=\sigma_{1}^{2}\left(\sigma_{1} \sigma_{2}^{-1}\right)^{k}$ gives the property for $c_{k}^{\prime}$ and $c_{k}^{\prime \prime}$ in (6.7). Considering $\beta \bar{\beta}=\left(\sigma_{1}^{l} \sigma_{2}^{-l}\right)^{k}$ gives a more general version of Proposition 6.14.

Theorem 6.15. Let $b_{0}=0, b_{1}=1$ and $b_{i}=b_{i-2}+l \cdot b_{i-1}$. Then,

$$
l\left(2 \sum_{i=1}^{k-1} b_{2 i}+b_{2 k}\right)
$$

is a square for $k$ odd. 
Considering 5-braids may give similar, however, less pleasant statements of this kind.

On the other hand, arithmetic results can have some knot theoretic consequences.

Corollary 6.16. Any rational knot with Conway notation $(1,1, \ldots, 1)$ ("twist plat knot" [27]) is not algebraically slice.

Proof. Use the result of [7] that no odd Fibonacci number $>1$ is a square. (As well-known, algebraically slice knots have square determinant.)

Remark 6.17. Of course, the same argument shows that this knot is not strongly +achiral, but this follows more generally for any rational knot from the result of Hartley-Kawauchi as the 2-branched cover homology group $H_{1}\left(D_{K}\right)$ is cyclic (and non-trivial), and hence not a double.

We can exploit the result of Hartley-Kawauchi more generally. Let $\phi_{\beta}$ be the linear operator

$$
S D_{n} \ni x \stackrel{\phi_{\beta}}{\longmapsto} x \prod_{j=1}^{k}\left(1+s_{i_{j}}\right) \in S D_{n}
$$

associated to $\beta=\prod_{j=1}^{k} \sigma_{i_{j}}^{(-1)^{i_{j}}} \in B_{n}$, with $S D_{n}$ and $s_{i}$ as given before the proof of Theorem 4.21. By writing out the endomorphisms $\phi_{\beta}$ as matrices, for appropriate $\beta$, we obtain squareness properties for some linear combinations of entries of such matrices.

Example 6.18. Consider the matrix

$$
A=\left(\begin{array}{rrrrr}
1 & 18 & 18 & 24 & 12 \\
0 & 13 & 0 & 18 & 0 \\
0 & 0 & 25 & 0 & 18 \\
0 & 18 & 0 & 25 & 0 \\
0 & 0 & 18 & 0 & 13
\end{array}\right)
$$

Then, writing $A^{k}=\left(a_{i, j}^{(k)}\right)_{i, j=1}^{5}$, we have that $a_{1,4}^{(2 k+1)}+a_{1,5}^{(2 k+1)}$ is always a square. This follows again from [21], as $A^{T}$ represents the endomorphism $\phi_{\beta}$ for $\beta=\sigma_{1} \sigma_{2}^{-2} \sigma_{1} \sigma_{2}^{-1} \sigma_{1}^{2} \sigma_{2}^{-1}$ in the basis (4.4) of $S D_{3}$. Interestingly, again 
$a_{1,4}^{(2 k)}+a_{1,5}^{(2 k)}$ is always of the form $10 x^{2}$, although there is no knot-theoretical explanation of this fact.

The last example, together with some further experiments, leads to the following conjecture.

Conjecture 6.19. If $\beta^{\prime} \in B_{3}$ is an alternating braid, and $\beta=\beta^{\prime} \overline{\beta^{\prime}}$, then $\phi_{\beta}$ Jordan-decomposes over the quadratic number field $\mathbb{Q}(\sqrt{d})$, or at least over $\mathbb{Q}(\sqrt{d}, i)$, where $i=\sqrt{-1}$ and $d=\operatorname{det}\left(\widehat{\beta^{2}}\right)$. Moreover, $\sqrt{\operatorname{det}\left(\widehat{\beta^{2 k}}\right) / d} \in \mathbb{Z}$ for all $k>0$.

As $S D_{n}$ has an antiautomorphism (turn around by $180^{\circ}$ ), for $\beta=\beta^{\prime} \overline{\beta^{\prime}}$, $\phi_{\beta}$ is conjugate to its inverse, so that the characteristic polynomial $\chi\left(\phi_{\beta}\right)$ of $\phi_{\beta}$ is self-conjugate, i.e., $\chi\left(\phi_{\beta}\right)(x) \sim \chi\left(\phi_{\beta}\right)\left(x^{-1}\right)$ (where $\sim$ denotes, as in definition 4.22 , equality up to units in $\left.\mathbb{Z}\left[x, x^{-1}\right]\right)$. However, $\chi\left(\phi_{\beta}\right)$ turns out to have (at least in all cases calculated in an experiment) some unexpected properties.

For 3-braids, the polynomial $\chi\left(\phi_{\beta}\right)$ had the form $(x-1) P(x)^{2}$ with a quadratic polynomial $P$, and in fact, $\phi_{\beta}$ decomposes into $I d_{1} \oplus \phi_{\beta}^{\prime} \oplus \phi_{\beta}^{\prime}$ (where $I d_{1}$ is the 1-dimensional identity map) under a certain, but not plausible, choice of basis.

For 5-braids $\chi\left(\phi_{\beta}\right)=(x-1)^{6} P_{1}(x)^{5} P_{2}(x)^{4}$ with $P_{1,2}$ being self-conjugate polynomials of degree 4 with alternating coefficients $\left(\left[P_{i}\right]_{x^{j}} \cdot\left[P_{i}\right]_{x^{j+1}}<0\right.$ for $0 \leq j<4)$, which additionally seem related, as always $\left[P_{1}\right]_{x}+\left[P_{2}\right]_{x^{2}}=+2$. For example, for

$$
\beta^{\prime}=\sigma_{3}^{-1} \sigma_{4} \sigma_{1}^{-1} \sigma_{2} \sigma_{4} \sigma_{1}^{-1} \sigma_{3}^{-1} \sigma_{2} \sigma_{4} \sigma_{3}^{-1}
$$

(and $\left.\beta=\beta^{\prime} \overline{\beta^{\prime}}\right)$ we have

$$
\begin{aligned}
\chi\left(\phi_{\beta}\right)= & (x-1)^{6}\left(1-26166 x+2297755 x^{2}-26166 x^{3}+x^{4}\right)^{5} \\
& \left(1-1533 x+26168 x^{2}-1533 x+x^{4}\right)^{4} .
\end{aligned}
$$

It is interesting to see what phenomena occur for more strands, but for 7-braids the dimension of $S D_{7}$ is 429 , and this renders experiments rather difficult.

These phenomena motivate and merit some possible further investigations in the future. 


\section{Acknowledgement.}

I would like to thank to M. Hirasawa, K. Murasugi, M. Thistlethwaite, K. Williams, D. Zagier, and the referee for some helpful remarks.

\section{References.}

[1] C. C. Adams, The knot book, W. H. Freeman \& Co., New York, 1994.

[2] J. W. Alexander, Topological invariants of knots and links, Trans. Amer. Math. Soc. 30 (1928), 275-306.

[3] D. Bar-Natan, S. Garoufalidis, L. Rozansky and D. P. Thurston, Wheels, Wheeling, and the Kontsevich Integral of the Unknot, Israel J. Math. 119 (2000), 217-237, see also q-alg/9703025.

[4] S. A. Bleiler, Realizing concordant polynomials with prime knots, Pacific J. Math. 100 (2) (1982), 249-257.

[5] G. Burde and H. Zieschang, Knots, de Gruyter, Berlin, 1986.

[6] T. D. Cochran and W. B. R. Lickorish, Unknotting information from 4-manifolds, Trans. Amer. Math. Soc. 297 (1) (1986), 125-142.

[7] J. H. E. Cohn, On square Fibonacci numbers, J. London Math. Soc. 39 (1964), 537-540.

[8] J. H. Conway, On enumeration of knots and links, in "Computational Problems in abstract algebra" (J. Leech, ed.), 329-358. Pergamon Press, 1969.

[9] R. Crowell, Nonalternating links, Illinois J. Math. 3 (1959), 101-120.

[10] O. T. Dasbach and S. Hougardy, A conjecture of Kauffman on amphicheiral alternating knots, J. Knot Theory Ramifications 5 (5) (1996), 629-635.

[11] J.-M. Deshouillers, F. Hennecart and B. Landreau, 7373170279850 , Math. Comp. 69 (229) (2000), 421-439.

[12] P. Di Francesco, O. Golinelli and E. Guitter, Meanders and the Temperley-Lieb algebra, Comm. Math. Phys. 186 (1) (1997), 1-59. 
[13] A. Di Porto and P. Filipponi, Some special triangular numbers, and recurring sequences, Notes Number Theory Discrete Math. 1 (1) (1995), $11-26$.

[14] A. Dujella, Diophantine quadruples for squares of Fibonacci and Lucas numbers, Portugal. Math. 52 (3) (1995), 305-318.

[15] A. Dujella, Generalized Fibonacci numbers and the problem of Diophantus, Fibonacci Quart. 34 (2) (1996), 164-175.

[16] A. Eswarathasan, On square pseudo-Lucas numbers, Canad. Math. Bull. 21 (3) (1978), 297-303.

[17] E. Flapan, A prime strongly positive amphicheiral knot which is not slice, Math. Proc. Cambridge Philos. Soc. 100 (3) (1986), 533-537.

[18] P. Freyd, J. Hoste, W. B. R. Lickorish, K. Millett, A. Ocneanu and D. Yetter, A new polynomial invariant of knots and links, Bull. Amer. Math. Soc. 12 (1985), 239-246.

[19] L. Goeritz, Knoten und quadratische Formen, Math. Z. 36 (1933), 647654 .

[20] G. H. Hardy and E. M. Wright, Einführung in die Zahlentheorie (German), R. Oldenbourg, Munich, 1958. (3rd edition)

[21] R. Hartley and A. Kawauchi, Polynomials of amphicheiral knots, Math. Ann. 243 (1) (1979), 63-70.

[22] C. Hooley, On Waring's problem, Acta Math. 157 (1-2) (1986), 49-97.

[23] J. Hoste and M. Thistlethwaite, KnotScape, a knot polynomial calculation and table access program, available at http://www. math.utk.edu/ ${ }^{\sim}$ morwen.

[24] V. F. R. Jones, A polynomial invariant of knots and links via von Neumann algebras, Bull. Amer. Math. Soc. 12 (1985), 103-111.

[25] V. F. R. Jones, Hecke algebra representations of of braid groups and link polynomials, Ann. of Math. 126 (1987), 335-388.

[26] V. F. R. Jones and J. H. Przytycki, Lissajous knots and billiard knots, "Knot theory" (Warsaw, 1995), Banach Center Publ. 42, Polish Acad. Sci., Warsaw, 1998, 145-163. 
Square numbers, spanning trees and invariants of achiral knots

[27] P. Jüde, 85 nützliche $\mathscr{G}$ dekorative Knoten, Weltbild Verlag, Augsburg, 1998.

[28] T. Kanenobu and H. Murakami, 2-bridge knots of unknotting number one, Proc. Amer. Math. Soc. 96 (3) (1986), 499-502.

[29] T. Kano, On the number of integers representable as the sum of two squares, J. Fac. Sci. Shinshu Univ. 4 (1969), 57-65.

[30] L. H. Kauffman, An invariant of regular isotopy, Trans. Amer. Math. Soc. 318 (1990), 417-471.

[31] L. H. Kauffman, State models and the Jones polynomial, Topology 26 (1987), 395-407.

[32] R. C. Kirby and W. B. R. Lickorish, Prime knots and concordance, Math. Proc. Cambridge Philos. Soc. 86 (3) (1979), 437-441.

[33] D. Krebes, An obstruction to embedding 4-tangles in links, Jour. of Knot Theory and its Ramifications 8 (3) (1999), 321-352.

[34] W. B. R. Lickorish, The unknotting number of a classical knot, in "Contemporary Mathematics" 44 (1985), 117-119.

[35] W. B. R. Lickorish, and K. C. Millett, A polynomial invariant for oriented links, Topology 26 (1) (1987), 107-141.

[36] W. B. R. Lickorish, and K. C. Millett, Some evaluations of link polynomials, Comment. Math. Helv. 61 (1986), 349-359.

[37] W. L. McDaniel, Diophantine representation of Lucas sequences, Fibonacci Quart. 33 (1) (1995), 59-63.

[38] W. W. Menasco, Closed incompressible surfaces in alternating knot and link complements, Topology 23 (1) (1986), 37-44.

[39] W. W. Menasco, and M. B. Thistlethwaite, The Tait flyping conjecture, Bull. Amer. Math. Soc. 25 (2) (1991), 403-412.

[40] J. Morgado, Note on the Chebyshev polynomials and applications to the Fibonacci numbers, Portugal. Math. 52 (3) (1995), 363-378.

[41] K. Murasugi, On a certain numerical invariant of link types, Trans. Amer. Math. Soc. 117 (1965), 387-422. 
[42] K. Murasugi, On periodic knots, Comment. Math. Helv. 46 (1971), $162-174$.

[43] K. Murasugi, Jones polynomial and classical conjectures in knot theory, Topology 26 (1987), 187-194.

[44] K. Murasugi, and A. Stoimenow, The Alexander polynomial of planar even valence graphs, Adv. Appl. Math. 31 (2) (2003), 440-462.

[45] B. R. Myers, On spanning trees, weighted compositions, Fibonacci numbers, and resistor networks, SIAM Rev. 17 (1975), 465-474.

[46] B. R. Myers, Number of spanning trees in a wheel, IEEE Trans Circuit Theory, 18 (1971), 280-282.

[47] C. V. Quach Hongler, On a theorem on partially summing tangles by Lickorish, Math. Proc. Cambridge Philos. Soc. 93 (1) (1983), 63-66.

[48] K. R. Rebman, The sequence: 151645121320 ... in combinatorics, Fib. Quart. 13 (1975), 51-55.

[49] D. Rolfsen, Knots and links, Publish or Perish, 1976.

[50] H. Schubert, Knoten mit zwei Brücken, Math. Z. 65 (1956), 133-170.

[51] N. J. A. Sloane, The On-Line Encyclopedia of Integer Sequences, accessible at the web address http://www.research.att.com/ njas/sequences/index.html.

[52] A. Stoimenow, Some minimal degree Vassiliev invariants not realizable by the HOMFLY and Kauffman polynomial, C. R. Acad. Bulgare Sci. 54 (4) (2001), 9-14.

[53] A. Stoimenow, On unknotting numbers and knot trivadjacency, partly joint with N. Askitas, Math. Scand. 94 (2) (2004), 227-248.

[54] A. Stoimenow, Polynomial values, the linking form and unknotting numbers, math.GT/0405076, Math. Res. Lett. 11 (5-6) (2004), 755769.

[55] A. Stoimenow, Generating functions, Fibonacci numbers, and rational knots, preprint math.GT/0210174.

[56] A. Stoimenow, Gauß sum invariants, Vassiliev invariants and braiding sequences, J. Of Knot Theory and Its Ram. 9 (2) (2000), 221-269. 
Square numbers, spanning trees and invariants of achiral knots

[57] A. Stoimenow, Jones polynomial, genus and weak genus of a knot, Ann. Fac. Sci. Toulouse VIII(4) (1999), 677-693.

[58] M. B. Thistlethwaite, A spanning tree expansion for the Jones polynomial, Topology 26 (1987), 297-309.

[59] D. Thoro, Two Fibonacci conjectures, Fibonacci Quart. 3 (1965), 184186.

[60] P. Traczyk, A criterion for signed unknotting number, Contemporary Mathematics 233 (1999), 215-220.

[61] K. Williams, Some refinements of an algorithm of Brillhart, Number theory (Halifax, NS, 1994), 409-416, CMS Conf. Proc. 15, Amer. Math. Soc., Providence, RI, 1995.

[62] C. C. Yalavigi, Two Fibonacci conjectures of Dmitri Thoro, Math. Education 5 (1971), A4.

[63] D. B. Zagier, Zetafunktionen und quadratische Körper, Eine Einführung in die höhere Zahlentheorie (German), Hochschultext. Springer-Verlag, Berlin-New York, 1981.

[64] D. B. Zagier, Modular forms of one variable, notes based on a course given in Utrecht, spring 1991.

[65] D. B. Zagier, A one-sentence proof that every prime $p \equiv 1(\bmod 4)$ is a sum of two squares, Amer. Math. Monthly 97 (2) (1990), 144.

Graduate School of Mathematical Sciences

UNIVERSITY OF TOKYO

3-8-1, КОМАВА, ТОКYO 153-8914

JAPAN

E-mail address: stoimeno@ms.u-tokyo.ac.jp

WWW: http://www.ms.u-tokyo.ac.jp/ stoimeno/

Received June 11, 2003. 